\title{
BASIC FLOWS OF GENERALIZED SECOND GRADE FLUIDS BASED ON A SISKO MODEL
}

\author{
A. WALICKA \\ University of Zielona Góra, Faculty of Mechanical Engineering \\ ul. Szafrana 4, 65-516 Zielona Góra, POLAND \\ E-mail: A.Walicka@ijame.uz.zgora.pl
}

\begin{abstract}
The present investigation is concerned with basic flows of generalized second grade fluids based on a Sisko fluid. After formulation of the general equations of motion three simple flows of viscoplastic fluids of a Sisko type or fluids similar to them are considered. These flows are: Poiseuille flow in a plane channel, Poiseuille flow in a circular pipe and rotating Couette flow between two coaxial cylinders. After presentation the Sisko model one was presented some models of fluids similar to this model. Next it was given the solutions of equations of motion for three flows mentioned above.
\end{abstract}

Key words: Sisko fluids, similar fluids, simple flows.

\section{Introduction}

In this paper we will consider the group of pseudoplastic fluids whose viscosity displays a non-linear relationship between the shear stress and the shear strain rate. Here the constitutive equations consider the shear stress as a non-linear function of the shear strain rate. One of more general models of this kind of fluids is a Sisko model $[1,2]$. There are a few simple solutions of the equations of motion for the flows of a Sisko fluid. The first were provided by $\mathrm{Na}$ and Hansen [3] and Bahrami et al. [4]; thereafter by Wang et al. [5], Hayat et al. [6], Khan et al. [7], Mekheimer and Kot [8], Khan and Shahzad [9], Akbar [10], Walicka [11].

The flows of fluids, whose models are similar to the Sisko model, were also studied by numerous rheologists; the researchers analysing the peristaltic flows should be mentioned here, for example: Akbar et al. [12] who analysed the flow of a Prandtl fluid, Nadeen [13] who analysed the flow of a tangent hyperbolic fluid model, Ellahi et al. [14] who analysed the flow of a Carreau fluid, etc.

In what follows we will present simple flows of generalized second grade fluids based on the Sisko model and similar models (see Table 1).

To this consider the other similar models of pseudoplastic fluid given in the second column of Tab.1. Note that for suitably selected material coefficients these models can be presented in a simple unified form

$$
\tau=\left(\mu_{0}+\mu_{i}|\dot{\gamma}|^{n_{i}}\right)
$$

To find three-dimensional forms of the stress tensor $\boldsymbol{T}$, corresponding to the above given onedimensional form of $\tau$ (called a constitutive relation), we may use the generalization of the Prager-Oldroyd method (Prager, [15]) applied to a Bingham fluid. This generalization can be found in (Walicka, [16, 17]) and its basis is as follows:

if

$$
\tau=f(\dot{\gamma}) \dot{\gamma},
$$

then 


$$
(\boldsymbol{T})_{i j}=T_{i j}=-p \delta_{i j}+2 f(A) D_{i j}=-p \delta_{i j}+f(A)\left(A_{l}\right)_{i j},
$$

where $p$ is the pressure and

$$
A=\left[\frac{1}{2} \operatorname{tr}\left(\boldsymbol{A}_{l}^{2}\right)\right]^{\frac{1}{2}}
$$

here $A$ is a square root from the second invariant of $\boldsymbol{A}_{1}$ [18].

\begin{tabular}{|c|c|c|c|c|}
\hline Author(s) & Model & Reduced model & $\mu_{i}$ & Comments \\
\hline \multicolumn{4}{|c|}{$n_{i}=n$} & $\begin{array}{c}\text { " } n+l " \\
\text { power models }\end{array}$ \\
\hline Sisko & $\tau=\left[\mu_{0}+\mu|\dot{\gamma}|^{n}\right] \dot{\gamma}$ & - & $\mu$ & \\
\hline $\begin{array}{l}\text { Carreau- } \\
\text { Yasuda }\end{array}$ & $\tau=\left\{\mu_{\infty}+\frac{\mu_{0}-\mu_{\infty}}{\left[1+(\kappa \dot{\gamma})^{n}\right]^{\frac{\alpha}{n}}}\right\} \dot{\gamma}$ & $\begin{array}{c}\tau \approx\left[\mu_{0}-\frac{\alpha \mu}{n}(\kappa \dot{\gamma})^{n}\right] \dot{\gamma} \\
\mu=\mu_{0}-\mu_{\infty}\end{array}$ & $-\frac{\alpha \mu \kappa^{n}}{n}$ & $\begin{array}{l}\text { Cross model for } \\
\quad \alpha=n \\
\text { Williamson- } \\
\text { Moore model for } \\
\quad \alpha=n=1\end{array}$ \\
\hline $\begin{array}{c}\text { Elsharkawy- } \\
\text { Hamrock }\end{array}$ & $\tau=\frac{\mu_{0} \dot{\gamma}}{\left[1+(\kappa \dot{\gamma})^{n}\right]^{\frac{1}{n}}}$ & $\tau \approx\left[\mu_{0}-\frac{\mu_{0}}{n}(\kappa \dot{\gamma})^{n}\right] \dot{\gamma}$ & $-\frac{\mu_{0} \kappa^{n}}{n}$ & \\
\hline \multicolumn{4}{|c|}{$n_{i}=2$} & "Cubic" models \\
\hline Prandtl & $\tau=\mu_{0} \frac{\arcsin (\kappa \dot{\gamma})}{(\kappa \dot{\gamma})} \dot{\gamma}$ & $\tau \approx\left[\mu_{0}+\frac{\mu_{0}}{\sigma}(\kappa \dot{\gamma})^{2}\right] \dot{\gamma}$ & $\frac{\mu_{0} \kappa^{2}}{6}$ & \\
\hline $\begin{array}{l}\text { Eyring- } \\
\text { Sutterby }\end{array}$ & $\tau=\mu_{0}\left[\frac{\operatorname{arsinh}(\kappa \dot{\gamma})}{\kappa \dot{\gamma}}\right]^{n} \dot{\gamma}$ & $\tau \approx\left[\mu_{0}-\frac{n \mu_{0}}{6}(\kappa \dot{\gamma})^{2}\right] \dot{\gamma}$ & $-\frac{n \mu_{0} \kappa^{2}}{6}$ & $\begin{array}{l}\text { Prandtl-Eyring } \\
\text { model for } n=1\end{array}$ \\
\hline Sutterby & $\begin{array}{l}\tau=\left\{\mu_{\infty}+\left(\mu_{0}-\mu_{\infty}\right) \times\right. \\
\left.\left[\frac{\operatorname{arsinh}(\kappa \dot{\gamma})}{\kappa \dot{\gamma}}\right]^{n}\right\} \dot{\gamma}\end{array}$ & $\begin{array}{c}\tau \approx\left[\mu_{0}+\frac{n \mu}{6}(\kappa \dot{\gamma})^{2}\right] \dot{\gamma} \\
\mu=\mu_{0}-\mu_{\infty}\end{array}$ & $-\frac{n \mu \kappa^{2}}{6}$ & $\begin{array}{l}\text { Powell-Eyring } \\
\text { model for } \\
n=1\end{array}$ \\
\hline $\begin{array}{l}\text { Gecim- } \\
\text { Winer }\end{array}$ & $\tau=\mu_{0}\left[\frac{\tanh (\kappa \dot{\gamma})}{(\kappa \dot{\gamma})}\right] \dot{\gamma}$ & $\tau \approx\left[\mu_{0}-\frac{\mu_{0}}{3}(\kappa \dot{\gamma})^{2}\right] \dot{\gamma}$ & $-\frac{\mu_{0} \kappa^{2}}{3}$ & \\
\hline \multicolumn{4}{|c|}{$n_{i}=1$} & $\begin{array}{c}\text { "Quadratic" } \\
\text { models } \\
\end{array}$ \\
\hline Bair-Winer & $\tau=\mu_{0}\left(\frac{1-e^{-\kappa \dot{\gamma}}}{\kappa \dot{\gamma}}\right) \dot{\gamma}$ & $\tau \approx\left[\mu_{0}-\frac{\mu_{0}}{2}(\kappa \dot{\gamma})\right] \dot{\gamma}$ & $-\frac{\mu_{0} \kappa}{3}$ & \\
\hline
\end{tabular}

Table.1. Model of fluids similar to the Sisko fluid model [18]. 


\section{Equations of motion of the unified Sisko fluid model}

The general equations of motion of a viscous fluid in a three-dimensional form are as follows:

- equation of continuity

$$
\operatorname{div} \mathbf{v}=0,
$$

- equation of momentum

$$
\rho \frac{d \mathbf{v}}{d t}=\operatorname{div} \boldsymbol{T}, \quad \boldsymbol{T}=-p \boldsymbol{1}+\boldsymbol{\Lambda}
$$

or

$$
\rho \frac{d \mathbf{v}}{d t}=-\nabla p+\operatorname{div} \boldsymbol{\Lambda}
$$

here

$$
\boldsymbol{\Lambda}=\left[\mu_{0}+\mu_{i}(A)^{n_{i}}\right] \boldsymbol{A}_{1}
$$

Many fluids of engineering interest appear to exhibit viscoelastic behaviour. Most popular are second grade fluids $[16 \div 20]$.

The constitutive relation for the second grade fluids is given as follows (Rivlin and Ericksen, [19])

$$
\boldsymbol{T}=-p \boldsymbol{I}+\mu \boldsymbol{A}_{1}+\alpha \boldsymbol{A}_{1}^{2}+\beta \boldsymbol{A}_{2}
$$

where $p$ is the pressure, $\mu$ is the coefficient of viscosity, $\alpha, \beta$ are material moduli, $\boldsymbol{A}_{1}$ and $\boldsymbol{A}_{2}$ are the first two Rivlin-Ericksen tensors defined by

$$
\begin{aligned}
& \boldsymbol{A}_{1}=\boldsymbol{2} \boldsymbol{D}=\boldsymbol{L}+\boldsymbol{L}^{T}, \quad \boldsymbol{A}_{1}^{2}=\boldsymbol{A}_{1} \cdot \boldsymbol{A}_{1}=\left(\boldsymbol{L}+\boldsymbol{L}^{T}\right)^{2}, \quad \boldsymbol{L}=\operatorname{grad} \mathbf{v}, \\
& \boldsymbol{A}_{2}=\dot{\boldsymbol{A}}_{1}+\boldsymbol{A}_{1} \boldsymbol{L}+\boldsymbol{L}^{T} \boldsymbol{A}_{1} \text { or } \boldsymbol{A}_{2}=\operatorname{grad} \boldsymbol{a}+(\operatorname{grad} \boldsymbol{a})^{T}+2 \boldsymbol{L}^{T} \boldsymbol{L}
\end{aligned}
$$

and

$$
\boldsymbol{a}=\frac{d \mathbf{v}}{d t}=\frac{\partial \mathbf{v}}{\partial t}+\mathbf{v} \boldsymbol{L}
$$

where $\mathbf{v}$ is the velocity vector, $\boldsymbol{a}$ is the acceleration vector and $($.$) represents the material derivative with$ respect to time.

To obtain a model that are does exhibit both pseudoplastic and viscoelastic behaviour we propose the following two constitutive equations for generalized second grade fluids (Walicki and Walicka, [20], Walicka $[16 \div 18])$ :

- for model I

$$
\boldsymbol{T}=-p \mathbf{1}+M_{i} \boldsymbol{A}_{1}+\alpha_{1} \boldsymbol{A}_{1}^{2}+\beta_{1} \boldsymbol{A}_{2},
$$


- for model II

$$
\boldsymbol{T}=-p \boldsymbol{1}+M_{i}\left(\boldsymbol{A}_{1}+\alpha_{2} \boldsymbol{A}_{1}^{2}+\beta_{2} \boldsymbol{A}_{2}\right),
$$

The viscosity function $M_{i}$ is given as follows:

$$
M_{i}=\mu_{0}+\mu_{i}(A)^{n_{i}}
$$

\section{Poiseuille flow in a plane channel}

Let us a consider the steady laminar fully developed flow of a generalized second grade Sisko fluid between two horizontal parallel plates (Fig.1). The flow takes place along to a pressure along the plates located at $y=-h$ and $y=+h$, respectively.

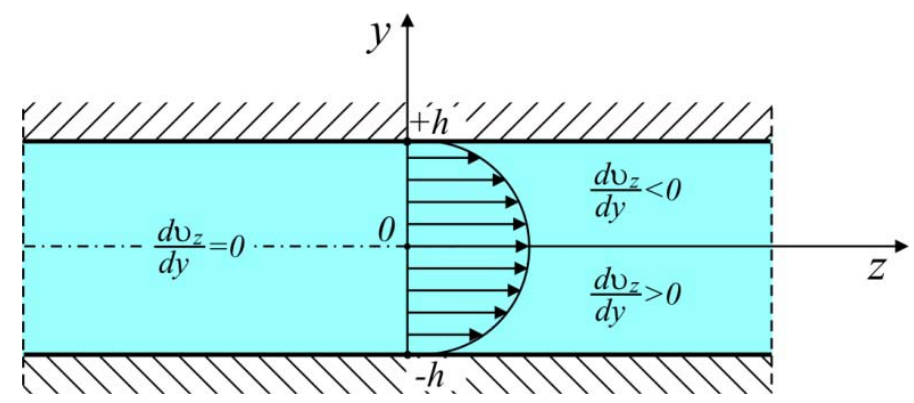

Fig.1. Channel between two parallel plates.

The flow field has the form

$$
\mathbf{v}=v_{z}(y) \boldsymbol{k}, \quad p=p(y, z) .
$$

Substituting Eqs (3.1) into Eq.(2.2), we obtain

$$
\begin{aligned}
& \frac{\partial}{\partial z}\left[-p+\alpha_{j} M_{i}^{j-1}\left(\frac{d v_{z}}{d y}\right)^{2}\right]+\frac{\partial}{\partial y}\left[M_{i} \frac{d v_{z}}{d y}\right]=0, \\
& \frac{\partial}{\partial y}\left[-p+\left(\alpha_{j}+2 \beta_{j}\right) M_{i}^{j-1}\left(\frac{d v_{z}}{d y}\right)^{2}\right]=0,
\end{aligned}
$$

where $j=1$ for model $I$ and $j=2$ for model $I I$.

Let us define a modified pressure $\hat{p}^{(j)}$ through

$$
\hat{p}^{(j)}=p-\left(\alpha_{j}+2 \beta_{j}\right) M_{i}^{j-1}\left(\frac{d v_{z}}{d y}\right)^{2},
$$

then from Eqs.(2.3) and (3.2), we obtain 


$$
\frac{d \hat{p}^{(j)}}{d z}=\frac{d \Lambda_{z y}}{d y}
$$

where

$$
\Lambda_{z y}=\left(\mu_{0}+\mu_{i}\left|\frac{d v_{z}}{d y}\right|^{n_{i}}\right) \frac{d v_{z}}{d y}
$$

Upon introducing Eq.(3.6) into Eq.(3.5), we have

$$
\frac{d \hat{p}^{(j)}}{d z}=\frac{d}{d y}\left[\left(\mu_{0}+\mu_{i}\left|\frac{d v_{z}}{d y}\right|^{n_{i}}\right) \frac{d v_{z}}{d y}\right]
$$

or

$$
\frac{1}{\mu_{0}} \frac{d \hat{p}^{(j)}}{d z}=\frac{d^{2} v_{z}}{d y^{2}}+\frac{\mu_{i}\left(n_{i}+1\right)}{\mu_{0}}\left|\frac{d v_{z}}{d y}\right|^{n_{i}} \frac{d^{2} v_{z}}{d y^{2}} .
$$

The boundary conditions on the plates are stated as follows

$$
\mathrm{v}_{z}=0 \quad \text { for } \quad y= \pm h
$$

Let us develop $v_{z}$ into a power series

$$
v_{z}=\beta^{0} v_{0}+\beta^{l} v_{1}+\beta^{2} v_{2}+\ldots
$$

where

$$
\beta=\frac{\mu_{i}\left(n_{i}+1\right)}{\mu_{0}}<1
$$

Putting Eq.(3.9) into Eq.(3.7) and retaining only the two first terms, we find

$$
\frac{1}{\mu_{0}} \frac{d \hat{p}^{(j)}}{d z}=\frac{d^{2} \mathrm{v}_{0}}{d y^{2}}+\beta\left[\frac{d^{2} \mathrm{v}_{1}}{d y^{2}}+\left(\left|\frac{d \mathrm{v}_{0}}{d y}\right|^{n_{i}}\right) \frac{d^{2} \mathrm{v}_{0}}{d y^{2}}\right] .
$$

After equating the like powers of $\beta$ we will obtain two equations:

- for $\beta^{0}$ :

$$
\frac{1}{\mu_{0}} \frac{d \hat{p}^{(j)}}{d z}=\frac{d^{2} \mathrm{v}_{0}}{d y^{2}}
$$


- for $\beta^{l}$ :

$$
\frac{d^{2} v_{1}}{d y^{2}}=-\left(\left|\frac{d v_{0}}{d y}\right|^{n_{i}}\right) \frac{d^{2} v_{0}}{d y^{2}}
$$

Solving these equations we will obtain

$$
\mathrm{v}_{0}=\frac{h^{2}-y^{2}}{2}\left(-\frac{1}{\mu_{0}} \frac{d \hat{p}^{(j)}}{d z}\right)
$$

and

$$
\mathrm{v}_{1}=\frac{h^{n_{i}+2}-|y|^{n_{i}+2}}{\left(n_{i}+1\right)\left(n_{i}+2\right)}\left(-\frac{1}{\mu_{0}} \frac{d \hat{p}^{(j)}}{d z}\right)^{n_{i}+1} .
$$

Finally:

$$
\mathrm{v}_{z}=\frac{h^{2}-y^{2}}{2}\left(-\frac{1}{\mu_{0}} \frac{d \hat{p}^{(j)}}{d z}\right)-\frac{\mu_{i}}{\mu_{0}} \frac{h^{n_{i}+2}-|y|^{n_{i}+2}}{\left(n_{i}+2\right)}\left(-\frac{1}{\mu_{0}} \frac{d \hat{p}^{(j)}}{d z}\right)^{n_{i}+1}
$$

The flow rate $Q$ is defined as

$$
Q=2 \int_{0}^{h} \mathrm{v}_{z} d y=\frac{3 h^{3}}{3}\left(-\frac{1}{\mu_{0}} \frac{d \hat{p}^{(j)}}{d z}\right)\left[1-\frac{3 \mu_{i} h^{n_{i}}}{\mu_{0}\left(n_{i}+3\right)}\left(-\frac{1}{\mu_{0}} \frac{d \hat{p}^{(j)}}{d z}\right)^{n_{i}}\right]
$$

Introducing the notation

$$
X=-\frac{1}{\mu_{0}} \frac{d \hat{p}^{(j)}}{d z}
$$

one can rewrite Eq.(3.17) in the form

$$
\frac{3 \mu_{i} h^{n_{i}}}{\mu_{0}\left(n_{i}+3\right)} X^{n_{i}+1}-X+\frac{3 Q}{2 h^{3}}=0 .
$$

Denoting its solution by $X_{s}$, we have

$$
\frac{d \hat{p}^{(j)}}{d z}=-\mu_{0} X_{s}
$$


hence

$$
\hat{p}^{(j)}=C-\mu_{0} X_{s} z
$$

and

$$
\frac{d \mathrm{v}_{z}}{d y}=-y X_{s}+\frac{\mu_{i}}{\mu_{0}}|y|^{n_{i}+1} X_{s}^{n_{i}+1}
$$

The final formula for the pressure distribution is as follows

$$
p=\hat{p}^{(j)}+\left(\alpha_{j}+2 \beta_{j}\right) M_{i}^{j-1}\left(\frac{d \mathrm{v}_{z}}{d y}\right)^{2}
$$

Note that for the regular Sisko fluid

$$
p=\hat{p}^{(j)} \text {. }
$$

\section{Poiseuille flow through a circular pipe}

Let us consider the steady laminar flow of a generalized second grade Sisko fluid in a circular pipe of radius $R$ (Fig.2). We are concerned about the velocity field in the form of:

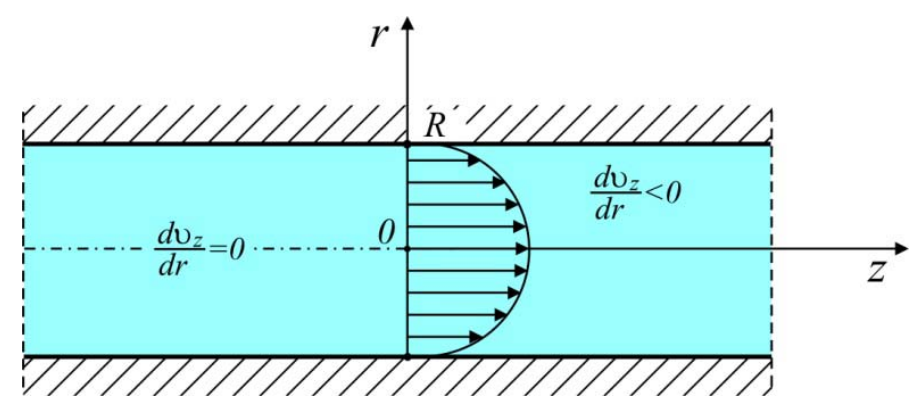

Fig.2. Geometry of a circular pipe.

$$
\mathrm{v}_{z}=0, \quad \mathrm{v}_{\vartheta}=0, \quad \mathrm{v}_{z}=\mathrm{v}_{z}(r)
$$

The equations of motion are now

$$
\begin{aligned}
& \frac{\partial p}{\partial r}=\left(\alpha_{j}+2 \beta_{j}\right) \frac{1}{r} \frac{d}{d r}\left[r M_{i}^{j-1}\left(\frac{d \mathrm{v}_{z}}{d r}\right)^{2}\right] \\
& \frac{\partial p}{\partial z}=\frac{1}{r} \frac{d}{d r}\left[r M_{i} \frac{d \mathrm{v}_{z}}{d r}\right]
\end{aligned}
$$

where $j=1$ for model $I$ and $j=2$ for model $I I$. 
Here we have

$$
M_{i}=\left[\mu_{0}+\mu_{i}\left(-\frac{d v_{z}}{d r}\right)^{n_{i}}\right]
$$

therefore Eq.(4.3) can be presented as

$$
\frac{\partial p}{\partial z}=\frac{1}{r} \frac{d\left(r \Lambda_{r z}\right)}{d r}
$$

where

$$
\Lambda_{r z}=M_{i} \frac{d v_{z}}{d r}
$$

hence

$$
\frac{r}{\mu_{0}} \frac{\partial p}{\partial z}=\frac{d}{d r}\left(r \frac{d v_{z}}{d r}\right)-\frac{\mu_{i}}{\mu_{0}} \frac{d}{d r}\left[r\left(-\frac{d v_{z}}{d r}\right)^{n_{i}+1}\right]
$$

The boundary conditions are

$$
\mathrm{v}_{z}=0, \quad \text { for } \quad r=R, \quad \frac{d \mathrm{v}_{z}}{d r}=0, \quad \text { for } \quad r=0
$$

Develop $v_{z}$ into a power series:

$$
\mathrm{v}_{z}=\mathrm{v}_{0}+\beta \mathrm{v}_{1}+\beta^{2} \mathrm{v}_{2}+\ldots
$$

where

$$
\beta=\frac{\mu_{i}}{\mu_{0}}
$$

and on introducing this series into Eq.(4.7), we will obtain for the two first powers of $\beta$ the following equations:

- for $\beta^{0}$

$$
\frac{d}{d r}\left(r \frac{d v_{0}}{d r}\right)=\frac{r}{\mu_{0}} \frac{\partial p}{\partial z}
$$

$-\quad$ for $\beta^{l}$

$$
\frac{d}{d r}\left(r \frac{d \mathrm{v}_{1}}{d r}\right)=\frac{d}{d r}\left[r\left(-\frac{d \mathrm{v}_{0}}{d y}\right)^{n_{i}+1}\right]
$$


Solving these equations we have, respectively

$$
\mathrm{v}_{0}=\frac{R^{2}-r^{2}}{4 \mu_{0}}\left(-\frac{\partial p}{\partial z}\right)
$$

and

$$
\mathrm{v}_{l}=-\frac{R^{n_{i}+2}-r^{n_{i}+2}}{\left(2 \mu_{0}\right)^{n_{i}+1}\left(n_{i}+2\right)}\left(-\frac{\partial p}{\partial z}\right)^{n_{i}+1}
$$

Finally, according to Eq.(4.9), there is

$$
\mathrm{v}_{z}=\frac{R^{2}-r^{2}}{4 \mu_{0}}\left(-\frac{\partial p}{\partial z}\right)-\frac{\mu_{i}}{\mu_{0}} \frac{R^{n_{i}+2}-r^{n_{i}+2}}{\left(2 \mu_{0}\right)^{n_{i}+1}\left(n_{i}+2\right)}\left(-\frac{\partial p}{\partial z}\right)^{n_{i}+1} .
$$

The flow rate is equal to

$$
Q=2 \pi \int_{0}^{R} v_{z}(r) r d r=\frac{\pi R^{4}}{4}\left(-\frac{1}{2 \mu_{0}} \frac{\partial p}{\partial z}\right)\left[1-\frac{4 \mu_{i} R^{n_{i}}}{\left(n_{i}+4\right)}\left(-\frac{1}{2 \mu_{0}} \frac{\partial p}{\partial z}\right)^{n_{i}}\right]
$$

Introducing the notation

$$
Y=-\frac{1}{2 \mu_{0}} \frac{\partial p}{\partial z}
$$

one can rewrite Eq.(4.16) in the form

$$
\frac{4 \mu_{i} R^{n_{i}}}{\mu_{0}\left(n_{i}+4\right)} Y^{n_{i}+1}-Y+\frac{4 Q}{\pi R^{4}}=0
$$

Denoting its solution by $Y_{s}$, we have

$$
\frac{\partial p}{\partial z}=-2 \mu_{0} Y_{s}
$$

and

$$
\frac{d v_{z}}{d r}=r Y_{s}\left[1+\left(\frac{\mu_{i}}{\mu_{0}}\right) r^{n_{i}} Y_{s}^{n_{i}}\right]
$$

The pressure distribution is now given as follows 


$$
p(r, z)=C-2 \mu_{0} Y_{s} z+\left(\alpha_{j}+2 \beta_{j}\right) \int \frac{1}{r} \frac{d}{d r}\left[r M_{i}^{j-1}\left(\frac{d \mathrm{v}_{z}}{d r}\right)^{2}\right] d r
$$

or

$$
p(r, z)=C-2 \mu_{0} Y_{s} z+\left(\alpha_{j}+2 \beta_{j}\right) J_{j}(r, z)
$$

where

$$
J_{l}(r, z)=\frac{3}{2}\left(r Y_{s}\right)^{2}+\frac{2\left(n_{i}+3\right)}{\left(n_{i}+2\right)}\left(\frac{\mu_{i}}{\mu_{0}}\right)\left(r Y_{s}\right)^{n_{i}+2}+\frac{2 n_{i}+3}{2 n_{i}+2}\left(\frac{\mu_{i}}{\mu_{0}}\right)^{2}\left(r Y_{s}\right)^{2 n_{i}+2}
$$

or

$$
J_{2}(r, z)=\mu_{0} J_{1}(r, z)+(-1)^{n_{i}+2} \mu_{i} \sum_{i=0}^{n_{i}+2} \frac{(i+1) n_{i}+3}{(i+1) n_{i}+2} C_{n_{i}+2}^{i}\left(\frac{\mu_{i}}{\mu_{0}}\right)^{i}\left(r Y_{s}\right)^{(i+1) n_{i}+2} .
$$

Note that for a regular Sisko fluid there is

$$
p=p(z)
$$

\section{Rotating Couette flow between two coaxial cylinders}

The fluid flow configuration is shown in Fig.3; the inner cylinder of radius $R_{i}$ rotates with a constant angular velocity $\omega$ and the outer cylinder of radius $R_{o}$ is fixed. The flow field of the fluid is given by

$$
\mathrm{v}_{r}=0, \quad \mathrm{v}_{\vartheta}=\mathrm{v}_{\vartheta}(r), \quad \mathrm{v}_{z}=0 .
$$

The equations of motion take the form

$$
\frac{d p}{d r}=\rho \frac{\mathrm{v}_{\vartheta}^{2}}{r}+\left(\alpha_{j}+2 \beta_{j}\right) \frac{d}{d r} M_{i}^{j-1}\left[r \frac{d}{d r}\left(\frac{\mathrm{v}_{\vartheta}}{r}\right)\right]^{2}+2 \beta_{j} \frac{M_{i}^{j-1}}{r}\left[r \frac{d}{d r}\left(\frac{\mathrm{v}_{\vartheta}}{r}\right)\right]^{2},
$$

here $j=1$ for model $I$ and $j=2$ for model $I I$;

$$
\frac{d}{d r}\left(r^{2} \Lambda_{r \vartheta}\right)=0
$$

where

$$
\Lambda_{r \vartheta}=M_{i}\left[r \frac{d}{d r}\left(\frac{\mathrm{v}_{\vartheta}}{r}\right)\right]
$$


and

$$
M_{i}=\mu_{0}+\mu_{i}\left[-r \frac{d}{d r}\left(\frac{\mathrm{v}_{\vartheta}}{r}\right)\right]^{n_{i}}
$$

finally,

$$
\Lambda_{r \vartheta}=\left\{\mu_{0}+\mu_{i}\left[-r \frac{d}{d r}\left(\frac{\mathrm{v}_{\vartheta}}{r}\right)\right]^{n_{i}}\right\}\left[r \frac{d}{d r}\left(\frac{\mathrm{v}_{\vartheta}}{r}\right)\right]
$$
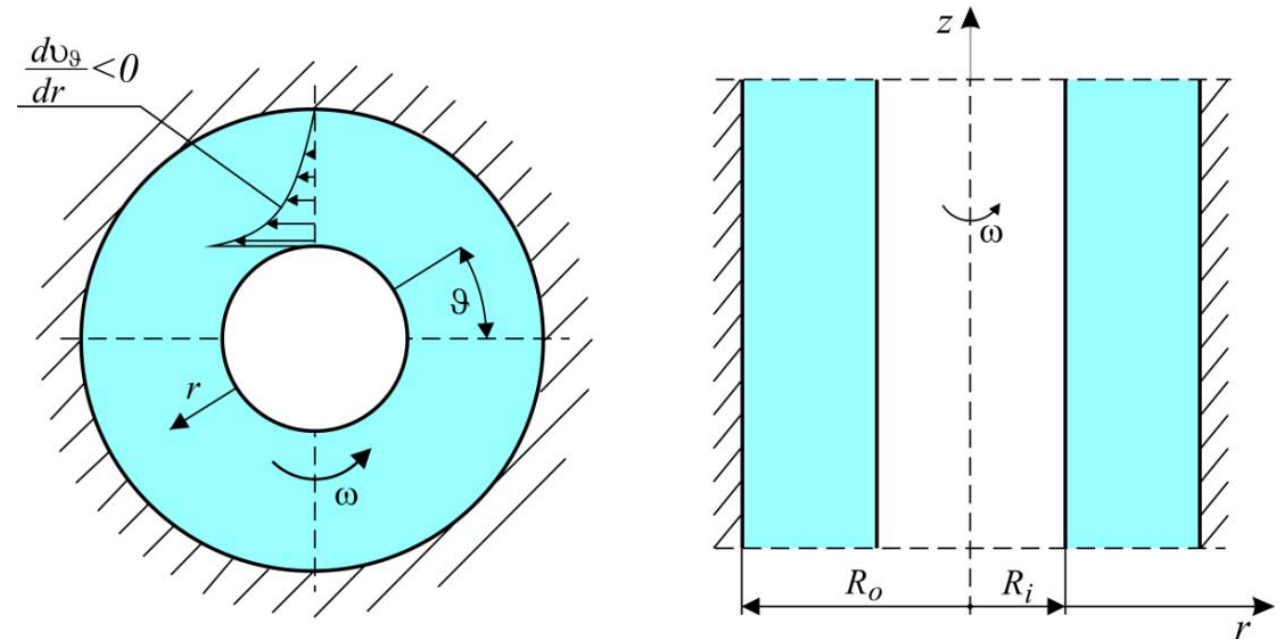

Fig.3. Geometry of the rotational flow between cylindrical surfaces.

The boundary conditions for velocity are

$$
\begin{aligned}
& \mathrm{v}_{\vartheta}=R_{i} \omega, \quad \text { for } \quad r=R_{i}, \\
& \mathrm{\cup}_{\vartheta}=0, \quad \text { for } \quad r=R_{o} .
\end{aligned}
$$

Upon integration of Eq.(5.3), we will obtain

$$
\Lambda_{r \vartheta}=\frac{C_{1}}{r^{2}}
$$

This result introduced into Eq.(5.6), will yield

$$
\frac{C_{1}}{\mu_{0} r^{2}}=r \frac{d}{d r}\left(\frac{\mathrm{v}_{\vartheta}}{r}\right)-\frac{\mu_{i}}{\mu_{0}}\left[-r \frac{d}{d r}\left(\frac{\mathrm{v}_{\vartheta}}{r}\right)\right]^{n_{i}+1} .
$$

To find the solution to this equation, develop the velocity $v_{\vartheta}$ into a series 


$$
v_{\vartheta}=v_{0}+\beta v_{1}+\beta^{2} v_{2}+\ldots
$$

where

$$
\beta=\frac{\mu_{i}}{\mu_{0}} .
$$

Putting now $v_{\vartheta}$ from Eq.(5.10) into Eq.(5.9) and retaining only the two first terms, we will obtain for the same powers of $\beta$ the following equations:

- for $\beta^{0}$

$$
\frac{C_{1}}{\mu_{0} r^{2}}=r \frac{d}{d r}\left(\frac{\mathrm{v}_{\vartheta}}{r}\right)
$$

- for $\beta^{l}$

$$
r \frac{d}{d r}\left(\frac{\mathrm{v}_{1}}{r}\right)=\left[-r \frac{d}{d r}\left(-\frac{\mathrm{v}_{0}}{r}\right)\right]^{n_{i}+1} .
$$

Upon solving these equations, we have

$$
\mathrm{v}_{0}=C_{2}^{(0)} r-\frac{C_{1}}{2 \mu_{0} r}
$$

and

$$
\mathrm{v}_{1}=C_{2}^{(1)} r-\frac{\left(-C_{1}\right)^{n_{i}+1}}{\mu_{0}^{n_{i}+1}\left(2 n_{i}+2\right) r^{2 n_{i}+1}}
$$

Finally, according to Eq.(5.10), we have

$$
\mathrm{v}_{\vartheta}=C_{2} r+\frac{\left(-C_{1}\right)}{2 \mu_{0} r}-\frac{\mu_{i}}{\mu_{0}} \frac{\left(-C_{1}\right)^{n_{i}+1}}{\mu_{0}^{n_{i}+1}\left(2 n_{i}+2\right) r^{2 n_{i}+1}} .
$$

Note that on the basis of the second boundary condition (5.7) we have

$$
C_{2}=-\frac{\left(-C_{1}\right)}{2 \mu_{0} R_{o}^{2}}+\frac{k_{i}}{\mu_{0}} \frac{\left(-C_{1}\right)^{n_{i}+1}}{\mu_{0}^{n_{i}+1}\left(2 n_{i}+2\right) R_{o}^{2 n_{i}+2}}
$$

and from the first boundary condition (5.6), we have 


$$
\omega=\frac{\left(-C_{1}\right)}{2 \mu_{0}}\left(\frac{1}{R_{i}^{2}}-\frac{1}{R_{o}^{2}}\right)+\frac{\mu_{i}}{\mu_{0}} \frac{\left(-C_{1}\right)^{n_{i}+1}}{\left(2 n_{i}+2\right)}\left(\frac{1}{R_{i}^{2 n_{i}+2}}-\frac{1}{R_{o}^{2 n_{i}+2}}\right) .
$$

The angular velocity $\omega(r)=\frac{v_{\vartheta}}{r}$ at any position $r$ is expressed as

$$
\omega(r)=C_{2}+\frac{\left(-C_{1}\right)}{2 \mu_{0} r^{2}}+\frac{\mu_{i}}{\mu_{0}} \frac{\left(-C_{1}\right)^{n_{i}+1}}{\mu_{0}^{n_{i}+1}\left(2 n_{i}+2\right) r^{2 n_{i}+2}},
$$

then

$$
\omega-\omega(r)=\frac{\left(-C_{1}\right)}{2 \mu_{0} r^{2}}\left(\frac{1}{\beta^{2}}-\frac{1}{\tilde{r}^{2}}\right)-\frac{\mu_{i}}{\mu_{0}} \frac{\left(-C_{1}\right)^{n_{i}+1}}{\mu_{0}^{n_{i}+1}\left(2 n_{i}+2\right) R_{o}^{2 n_{i}+2}}\left(\frac{1}{\beta^{2 n_{i}+2}}-\frac{1}{\tilde{r}^{2 n_{i}+2}}\right),
$$

where

$$
\beta=\frac{R_{i}}{R_{o}}, \quad \tilde{r}=\frac{r}{R_{o}} .
$$

The unit torque acting on the cylindrical surface of radius $r$ is equal to

$$
T=2 \pi r^{2} \Lambda_{r \vartheta}=2 \pi C_{1} .
$$

Denoting, respectively, by $T_{s}$ the torque acting on the inner cylinder to maintain its motion and by $T_{r}$ the anti-torque applied to the outer cylinder to maintain its rest we have

$$
\begin{aligned}
& T=\left\{\begin{array}{l}
T_{r}=-2 \pi C_{1}, \\
T_{s}=2 \pi C_{1},
\end{array}\right. \\
& \omega-\omega(r)=\frac{1}{2 \mu_{0}}\left(\frac{T}{2 \pi R_{o}^{2}}\right)\left[\left(\frac{1}{\beta^{2}}-\frac{1}{\tilde{r}^{2}}\right)+\right. \\
& \left.-\frac{\mu_{i}}{\mu_{0}}\left(\frac{T}{2 \pi R_{o}^{2}}\right)^{n_{i}} \frac{1}{\mu_{0}^{n_{i}}\left(n_{i}+1\right)}\left(\frac{1}{\beta^{2 n_{i}+2}}-\frac{1}{\tilde{r}^{2 n_{i}+2}}\right)\right]
\end{aligned}
$$

the formula which can be used for determining the material constants in the Sisko model from measurements of torque and angular velocity in a coaxial annular viscosimeter [21].

\section{Conclusions}

Simple flows of generalized second grade pseudoplastic fluids based on Sisko model or similar models may find many applications in a different branches of technology and industry. It can cite for example the theory of lubrication or petrochemical technology. Basing on the general equations of motions 
of the Sisko model of fluid it was presented equations of motion for thee flows, namely two Poiseuille flows in: plane channel, circular tube, and rotating Couette flow between two coaxial cylinders. The given solutions to Poiseuille flows may be used to modelling the flows in geological beds or porous layers whereas the Couette flow can serve to measuring the physical fluids parameters.

\section{Nomenclature}

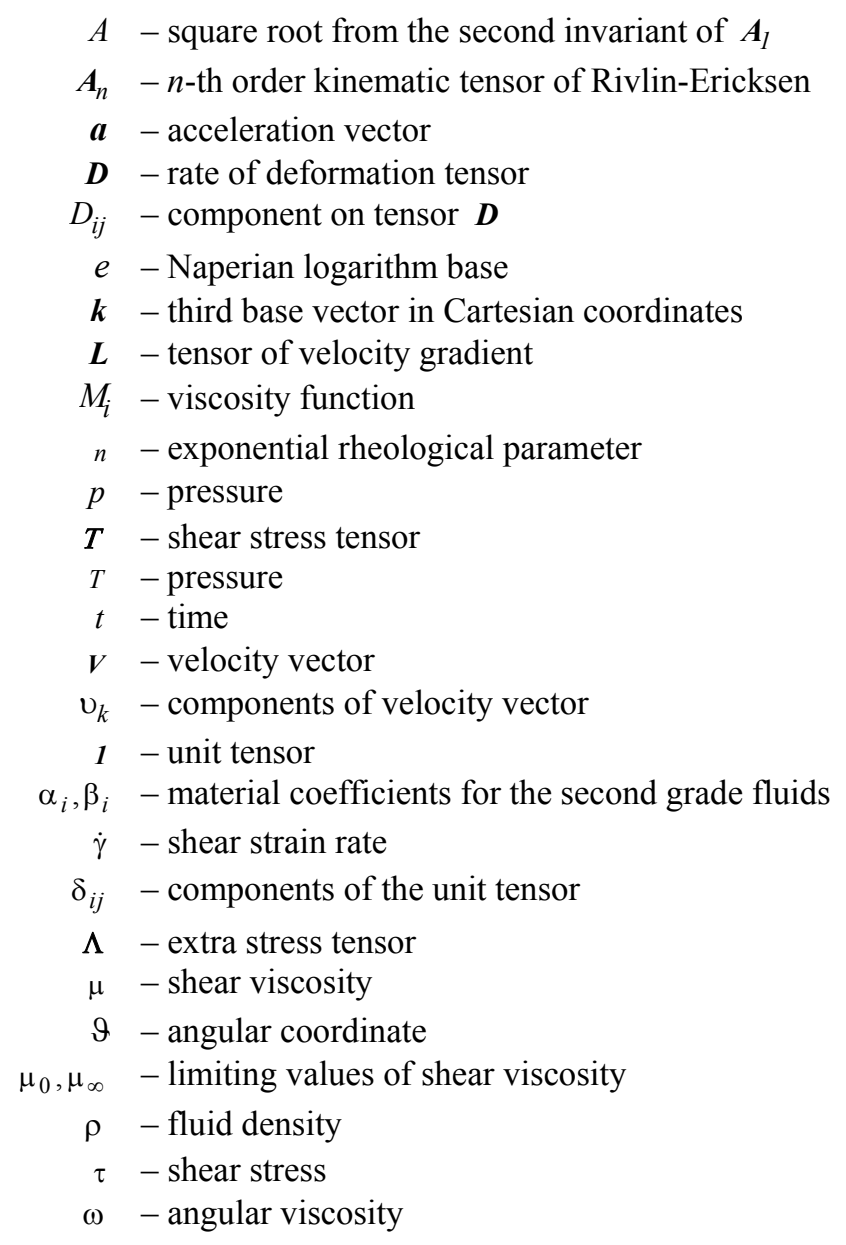

\section{References}

[1] Sisko A.W. (1958): The flow of lubricating greases. - Indust. Eng. Chemistry, vol.50, No.12, pp.1789-1792.

[2] Sisko A.W. (1960): Capillary viscometer for non-Newtonian liquids. - J. Colloid Sci., vol.15, No.1, pp.89-96.

[3] Na T.Y. and Hansen A.G. (1967): Radial flow of viscous non-Newtonian fluids between disks. - Int. J. Non-Linear Mechanics, vol.2, No.3, pp.261-273.

[4] Bahrami A., Azizmohhmmadi S., Narenji M.R.G. and Taghikhani V. (1996): Isothermal axial laminar flow of non-Newtonian fluids in concentric annuli (power-law and Sisko fluids). - Iranian Polym. J., vol.5, No.4, pp.271-278.

[5] Wang Y., Hayat T. and Ali N., Oberlack M. (2008): Magnetohydrodynamic peristaltic motion of a Sisko fluid in a symmetric or asymmetric channel. - Physica A, vol.387, pp.347-362. 
[6] Hayat T., Maqbool K. and Asghar S. (2009): Hall and heat transfer effects on the steady flow of a Sisko fluid. Z.Naturforsch. vol.64a, pp.769-782.

[7] Khan M., Munawar S. and Abbasbandy S. (2010): Steady flow and heat transfer of a Sisko fluid in annular pipe. Int. J. Heat Mass Transfer, vol.53, pp.1290-1297.

[8] Mekheimer Kh. S. and El Kot M.A. (2012): Mathematical modelling of unsteady flow of a Sisko fluid through an anisotropically tapered elastic arteries with time-variant overlapping stenosis. - Appl. Math. Modelling. vol.36, No.11, pp.5393-5407.

[9] Khan M. and Shahzad A. (2012): On axisymmetric flow of Sisko fluid over a radially stretching sheet. - Int. J. NonLinear Mech., vol.47, pp.999-1007.

[10] Akbar N.S. (2014): Peristaltic Sisko nano-fluid in an asymmetric channel. - Appl. Nanosci., vol.4, pp.663-673, DOI: $10.1007 / \mathrm{s} 13204-013-0205-1$.

[11] Walicka A. (2016): Curvilinear squeeze film bearing lubricated by a Sisko fluid or by one of similar fluids. submitted to: Chem. Proc. Eng.

[12] Akbar N.S., Nadeem S. and Lee C. (2012): Peristaltic flow of a Prandtl fluid model in an asymmetric channel. Int. J. Phys. Sci., vol.7, No.5, pp.687-695.

[13] Nadeem S., Sadaf H. and Akbar N.S. (2015): Effects of nanoparticles on the peristaltic motion of tangent hyperbolic fluid model in an annulus. - Alexandria Eng. J., vol.54, pp.843-851.

[14] Ellahi R., Riaz A., Nadeem S. and Ali M. (2012): Peristaltic flow of Carreau fluid in a rectangular duct through a porous medium. - Math. Problems in Eng., vol.2012, Article ID 329639, p.24.

[15] Prager W. (1961): Introduction to Mechanics of Continua. - Boston: Ginn.

[16] Walicka A. (2002): Rheodynamics of Non-Newtonian Fluids Flow in Straight and Curved Channels. (in Polish). Zielona Gora: University Press.

[17] Walicka A. (2002): Rotational Flows of the Rheologically Complex Media in Thin Annular Channels. (in Russian). - Zielona Gora: University Press.

[18] Walicka A. (2017): Rheology of fluids in Mechanical Engineering. - Zielona Góra: University Press.

[19] Rivlin R.S. and Ericksen J.L. (1955): Stress deformation relations for isotropic materials. - J. Rat. Mech. Anal., vol.4, No.4, pp.323-425.

[20] Walicki E. and Walicka A. (1998): Basic flows of generalized second grade fluids. - XIX Symposium on Rheology, Klaipeda, Collection of Abstracts, p.78.

[21] Schramm G. (1994): A Practical Approach to Rheology and Rheometry. - Karlsruhe: Haake,.

Received: June 12, 2017

Revised: June 29, 2017 